\title{
Creating an Interactive and Storytelling Educational Physics App for Mobile Devices
}

\author{
Krzysztof Szklanny, Łukasz Homoncik, Marcin Wichrowski, Alicja Wieczorkowska \\ Polish-Japanese Academy of Information Technology, Koszykowa 86, Warsaw, Poland \\ Email: \{kszklanny, lukasz.homoncik,mati\}@pjwstk.edu.pl, alicja@poljap.edu.pl
}

\begin{abstract}
Ubiquitous mobile technology makes m-learning a popular method of education. Our goal was to use education and entertainment (edutainment) for teaching physics on mobile devices, as the technology is available for most of the students. A game was designed, as an interactive and storytelling educational physics app based on user experience and edutainment guidelines. The testers in usability tests considered the game useful; they also suggested improvements. Learning through play can be broadly applied to teach physics, educational apps can be created, and supplement education.
\end{abstract}

\section{INTRODUCTION}

$\mathrm{M}$ -LEARNING is a teaching technique that requires the use of portable devices (mobile phones, smartphones, PDAs, tablets) [1]; it may take place outside of school [2]. It is distance learning based on mobile phones, smartphones, PDAs and tablets with wireless Internet, with communication replaced with various communication technologies.

"Edutainment" (education and entertainment) is applied in m-learning. It encourages the user to learn through games and interaction, and evokes emotions, so it is easier to remember the content. Entertainment is (9P): Perennial, Pervasive, Popular, Personal, Pleasurable, Persuasive, Passionate, Profitable, and Practical [3]. Learning in the $21^{\text {st }}$ century is: Learner-centered, Media-driven, Personalized, Transfer-by-Design, Visibility Relevant, Data-Reach, Adaptable, Interdependent, and Diverse (9 features) [4].

The effectiveness of edutainment has been proven [5], [6]; it gives the opportunity to interact, for example in a game.

Edutainment has spread with the development of mobile devices (more common now than desktops). Smartphones sales increase by $70-80 \%$ per year [7]. Since 2013 more smartphones than feature phones are sold, and 50\% of people own a mobile phone. Various apps increased the capabilities of smartphones, especially with Internet access. The number of Internet surfers exceeds 3.6 billion [8]

Our app Apollo is a form of edutainment. It combines a game and a multimedia presentation, using the methodology proposed by the authors, including interaction, storytelling and a 3D (3-dimensional) visualization. It presents physics concepts, including satellite motions, geostationary orbit and gravitational acceleration [9]. Our goal was to visualize the

This work was partially supported by the Research Centre of the PolishJapanese Academy of Information Technology, supported by the Ministry of Science and Higher Education in Poland phenomena of physics and present them in an entertaining way. Physics is a difficult class; teachers indicate that young people lose the ability to imagine abstract concepts, and have difficulties in understanding the physics laws. There are not many physics apps available on the market.

The app was prepared in Polish, for junior high school students, who spend a lot of time on their smartphones. We researched on market penetration with smartphones, and checked which operating system is most popular. The usability tests of the app are presented in this paper.

\section{MOBILE MARKET ANALYSIS}

\section{A. World}

More than 1.2 billion smartphones were sold worldwide in 2014[10], and over 1.4 billion in 2016. Currently, 4.6 billion mobile devices are registered, and there will be more active devices than people [11]. In 2016, mobile web usage overtook desktop [12]. $80 \%$ of internet users own a smartphone [13]. The interest in edutainment also grows.

The most popular brand of mobile devices is Samsung [14], with Android as the most popular operating system [7]. Young people 18-30, often referred to as 'Generation Y', mostly use Apple or Samsung devices [15]. They live with phones in their hands. $60 \%$ permanently participate in the life of social networks, and browse news. $70 \%$ cannot imagine their lives without mobile apps and use 1-9 apps every day. This brings an opportunity for m-learning, and we decided to create the app for Android-based smartphones.

\section{B. Predictions for the Future}

Customizing websites for mobile devices will become increasingly important. Mobile devices are equipped with gyroscopes, GPS, multi-touch technology, accelerometers and cameras, which increases their potential for edutainment.

By 2020 mobile devices with the latest technologies available today will cost $\$ 10$ [16]. Paper will be replaced by mobile devices [17]; a digital notebook that allows displaying content is currently sold for $\$ 100$ [18].

Mobile devices are becoming thinner, of credit card size, and flexible [19]. It will be possible to adjust mobile devices to any shape [20]; they are already produced as bracelets. The devices will be multisensory, allowing for detection and emission of smells. Mobile device sensors that monitor heartrate are already available. We will be able to monitor 
the body state in real time, with high-speed wireless access to the cloud computing. Monitoring tools, wearable devices, mobile User Experience (UX) design, and location sensing are already the most significant mobile technologies [21]. Works on better batteries are also performed.

The research on mobile devices continues. Toyota's Windows to World based on AR (Augmented Reality) turns the car window into an interactive multi-touch screen, which can also be used as a drawing tool that references real world objects; work is underway on future cars that communicate with each other. The University of Washington is working on transparent AR contact lenses powered by solar energy.

\section{Poland}

The number of mobile app users keeps growing; $44 \%$ of mobile phone users owned a smartphone in 2014 , but $1 / 3$ of them do not buy apps from app stores [22]. They use phones for Internet browsing (mostly to use social media), watch video, play mobile games, as localizers, and for banking. But, $74 \%$ of $15-19$ year-old teens own a smartphone [23].

Daily Internet usage rate in Poland in 2016 was $90 \%$ in the age group 16-24 [10], with the average Internet speed 14 Mbps for download and 4.9 Mbps for upload [24].

\section{Mobile apps market}

Google Play has 2.8 million apps for Android, App Store has 2 million apps for iOS. Every month 100,000 apps are introduced. In 2014, 179 billion apps were downloaded. $11 \%$ of smartphone and tablet users have educational apps installed [15]. Entertainment apps are most often downloaded. In Poland, most common are instant messaging and social networking apps, games, radio and navigation.

\section{PHYSICS APPS FOR SMARTPHONES}

14 apps are listed at [25]; 6 are marked with 4 (out of 5) stars: VMS-Velocity and Acceleration Animation, Newtonium-Physics Simulator, Bridge Constructor Playground, Thomas Edison, Tory Odyssey: Motion Commotion, and Physics One Gravity.

$$
\begin{aligned}
& \text { 2. FORCES } \\
& \text { ATTRACTIVE FORCE } \\
& \text { m1, m2: mass of } 2 \text { objects; r: distance between } 2 \\
& \text { objects; G: gravitational constant }
\end{aligned}
$$

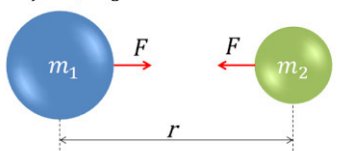

Figure 1. Physics Formulas Free app (screen from Google Play)

At Google Play, there are several hundred apps for physics, with several dozen for learning, mostly in English:

- Physics Formulas Free, rated 4.5 (out of 5) stars, with more than 500,000 downloads,

- Complete Physics, rated 4.1, over 500,000 downloads,

- Learn Physics, rated 4 stars, over 500,000 downloads,

- PhyWiz-Physics Solver, 4.6, over 100,000 downloads,

- Physics Notes, rated 4.2 stars, over 100,000 downloads.

\section{Physics Lesson}

The diagram below shows a graphical

representation of simple harmonic

motion.

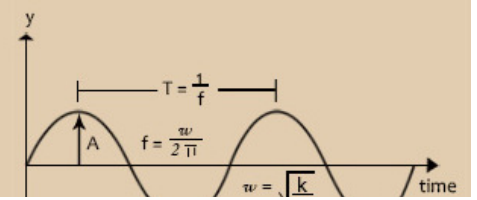

Figure 2. Complete Physics app (screen from Google Play)

Physics Formulas Free (Figure 1) is for calculating physics problems; it allows adding user-defined formulas. Complete Physics (Figure 2) has tutorials, questions, and a quiz. Learn Physics (Figure 3) has tutorials, formulas calculator and quizzes. PhyWiz - Physics Solver helps doing physics homework; it solves physics questions. Physics Notes has tutorials, step by step instructions, and allows learning in a deep and intuitive way. It is praised [25], but criticized: "I would like to see (...) testing features and instant-feedback in order to really put the theory into practice. (...) it would be great if students could add their own notes (...) it will enhance the revision."

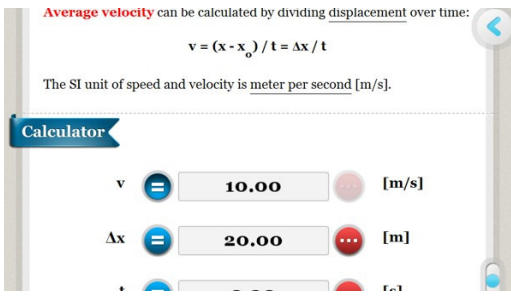

Figure 3. Learn Physics app (screen from Google Play)

\section{A. Physics Apps in Polish}

There are few apps for learning physics in Polish, not offering entertainment. Fizyka na 5! (Figure 4), rated 4.1, with over 1,000,000 downloads is a a crib with formulas. Fizyka-Stownik, rated 4.4, 50,000-100,000 downloads, is a physics dictionary, with quizzes. Fizyka - kalkulator, rated $4.3,10,000-50,000$ downloads, calculates physical formulas.

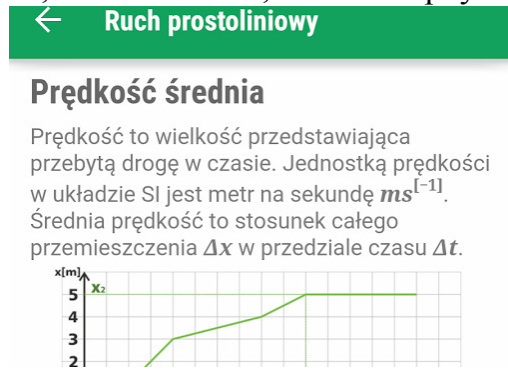

Figure 4 Fizyka na 5! app (screen from Google Play)

\section{APOLLO APP}

Based on the analysis of physics apps, mobile devices market, and operating systems, we prepared an edutainment physics app for Android. We used our experience in implementing an educational platform with elements of puzzle learning [26]. The app, Apollo (Figure 5) is a RPG 
(role playing game), with storytelling and 3D world [9] in the first person perspective - rendered from the player's viewpoint.

\section{A. Storytelling}

In interactive storytelling, the user can control the course of events in an app. It can be applied to physics education [27], [28], but there are no such games on the Polish market.

The narrative of the Apollo app is based on space travel. The spaceship Apollo experiences propulsion breakdown and loses contact with Earth. The computer must be fixed, and to do this, it is necessary to contact Earth. The pilot must help in determining the parameters necessary to send the message, and the computer gives the user a physics task to solve, and shows an explanatory video presentation.

\section{B. Interactive $3 D$ World}

The most common engine for creating games is Unity 3D [29]; also Unreal Engine [30] is popular. We used Unity 4.5. Unity works with Direct 3D (for Windows), and OpenGL ES for Android. It offers options for real time rendering, and detects the best hardware settings of a device on which the app is initialized, and automatically adjusts the settings. Functionality tests were carried out in Unity 3D Remote; simulating the game on the target device.

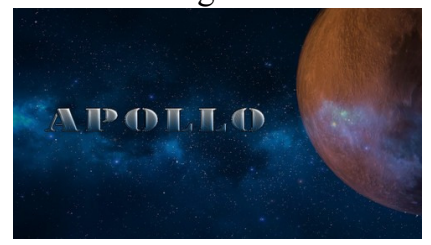

Figure 5 Start screen of the Apollo app

\section{User Interface and Interaction}

The app should be easy to use for junior high school students, so we followed UX guidelines [6]. The game is controlled through gestures, 2 virtual joysticks displayed on the screen, and a touch-activated interface of high usability. Our app is engaging, and uses simple communication.

The user of the Apollo app moves in a space station (Figure 6) using virtual joysticks in the lower corners of the screen. Consequent steps encourage the user to learn. The user explores the spaceship, to find hints and educational materials on the guidance computers. The interaction is through touching or approaching an interactive object (Figure 7), which have charts with formulas (Figure 8), movies, etc.

\section{Implementation}

The app is for Android 2.3.1 or higher, GPU supporting OpenGLES 2.0 or higher, Internet, and 1 GB of RAM.

The planet and spaceship were modelled in 3ds Max [31]. Animation was rendered using V-Ray 2.0, and 3D objects exported to FBX format. Textures, texts and graphics (1024 $x 1024)$ were made in Adobe Photoshop [32], and normal maps in Quixel Suite [33]. 25 colliders with an active trigger function were designed to activate sliding doors animations, move consoles, and to activate video clips and sounds.

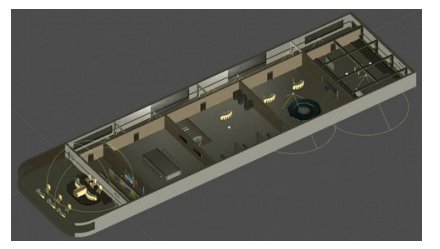

Figure 6 The interior of the space ship in Apollo, with lights shown

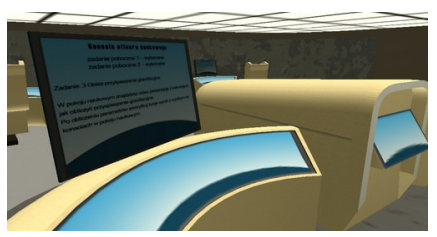

Figure 7 Interactive element in Apollo: the screen

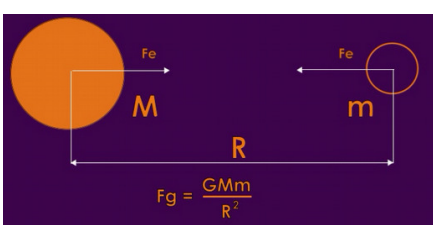

Figure 8 A chart with physical formulas in Apollo

We used speech synthesis [34] to get the voice of the computer. Adobe Audition [32] was used to edit audio files. Intro was created in Adobe After Effects [32].

\section{V.UsABILITY TESTS OF APOLLO}

The usability tests were performed using Samsung Galaxy Tab 4 (10" screen), to observe how the users navigate the game environment and accomplish simple tasks in their first contact with the app. We also wanted to study the users' first impression of educational storytelling on a mobile device.

11 IT students (Information technology, college seniors from Poland, age: $22-46, \mathrm{M}=29.9, \mathrm{SD}=7.6$ ) took part in the experiments; 5 participants is sufficient in such tests [35]. All testers used tablets and smartphones before. Each student owned a smartphone and 8 of them also a tablet. All except 1 person had already used educational apps:

- 7 persons used educational applications on laptops,

- 6 used educational apps on smartphones,

- 5 used educational applications on desktop computers,

- 4 used educational apps on tablets,

- 1 used educational games on Nintendo DS console.

Prior experience of the students included: foreign language learning (7 persons), programming language learning ( 2 persons), and geography and anatomy learning (one person each). 1 person never used educational apps.

\section{A. Test Procedure}

Each test had a form of an individual session, guided by a moderator, observing the user performing the tasks:

1. Start the app on the tablet.

2. Start navigating a character in the game environment.

3. Leave a room and go to the bridge deck of the space ship.

4. Find the captain's console; listen to the computer's orders.

5. Find the navigation console with further instructions.

6. Find the science room and see an educational video. 
Next, the testers completed an online survey, to assess the usability, interaction and audio-visual aspects of the app, and prior experience in using mobile devices in education.

\section{REsults OF UsABILITy TESTS}

\section{To what extent the app was easy to use?}

A majority of users (Figure 9) evaluated the app as easy to use $(\mathrm{M}=3.8, \mathrm{SD}=0.6 ; \mathrm{M}-$ mean value, $\mathrm{SD}-$ standard deviation).

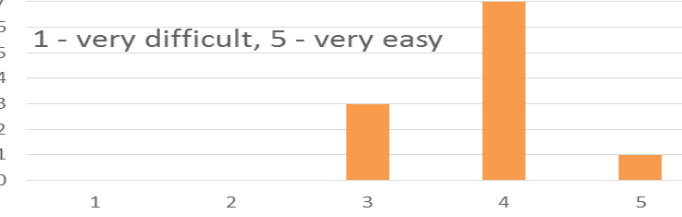

Figure 9 Ease of use of the Apollo app

2. To what extent it was easy to navigate the character?

The users evaluated it as convenient $(\mathrm{M}=3.6, \mathrm{SD}=0.9$,

Figure 10). Some users had difficulties to navigate the characters at the beginning. They were unable to find controllers, shown as semi-transparent ellipses in the corners (Figure 11).

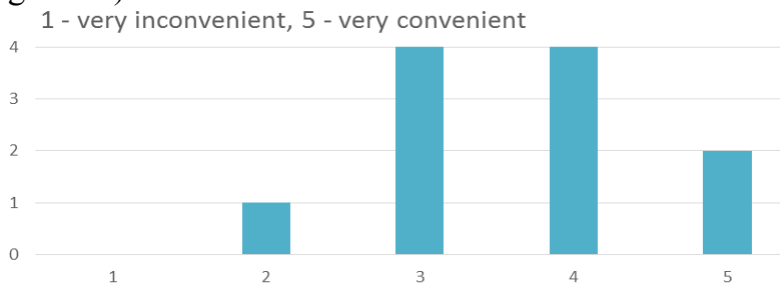

Figure 10 Convenience of navigating the character in the app

\section{To what extent the graphical elements of the interface} were clear and understandable?

They were mostly evaluated as understandable and very understandable (Figure 12, $\mathrm{M}=3.8, \mathrm{SD}=1.1$ ). The users tested if it was easy to find infographics, recognize them as navigation elements, and follow. Some users missed lettering on the doors, and readability of information screens was low in some cases because of poorly selected colours and fonts.

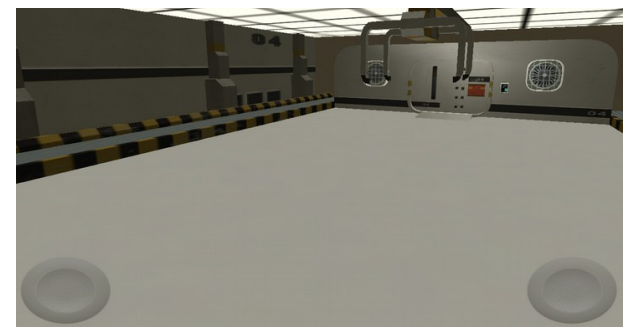

Figure 11 Controllers in Apollo (in the lower corners)

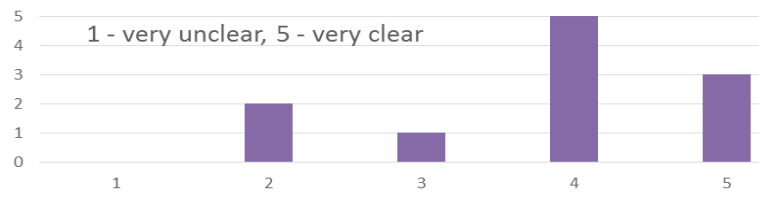

Figure 12 Understandability of the graphical elements of the interface
4. Was the size of buttons appropriate?

All users considered it appropriate $(\mathrm{M}=3.0, \mathrm{SD}=0.0)$.

5. Was the size of fonts and graphics large enough to maintain their readability?

Opinions (Figure 13) were mainly moderate and very good $(\mathrm{M}=3.6, \mathrm{SD}=1.2)$. Some users did not notice letterings on the door, and readability of information screens was also low in some cases because of poorly selected colours and font. Therefore, colours and fonts should be improved.

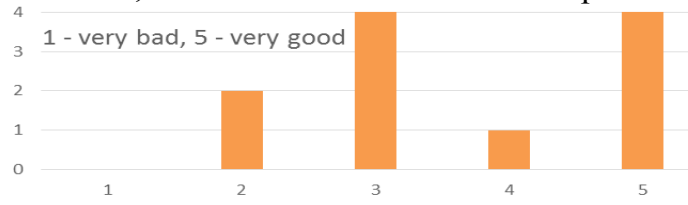

Figure 13 Size of fonts and graphics in the app

\section{Evaluation of scoring}

Most users evaluated scoring and sound quality as good and very good, but some as moderate or bad $(\mathrm{M}=3.8$, $\mathrm{SD}=1.2$, Figure 14). The users mainly complained about the bothersome voice of the synthesizer, reading the commands.

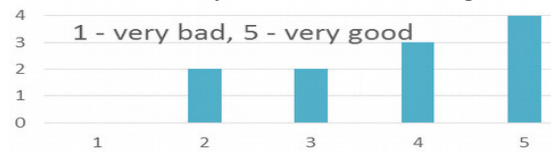

Figure 14 Evaluation of scoring in the app

\section{Aesthetic value of the app}

Diverse opinions were shown $(\mathrm{M}=3.6, \mathrm{SD}=1.1$, Figure 15). Probably more sophisticated design is needed.

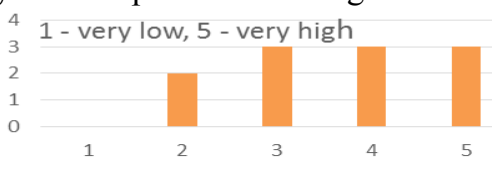

Figure 15 Aesthetic value of the app

8. General evaluation of the app for tablets

Most users evaluated the app for tablets as moderate or good to be used on tablets $(\mathrm{M}=3.5, \mathrm{SD}=0.8$, Figure 16).

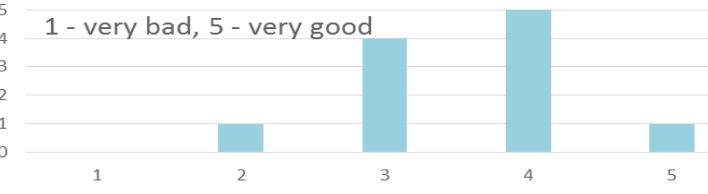

Figure 16 General evaluation of the app for tablets

9. How do you evaluate the usefulness of similar educational apps for learning with tablets?

Most users evaluated it as useful, but some might had a bad experience $(\mathrm{M}=3.7, \mathrm{SD}=1.5$, Figure 17$)$.

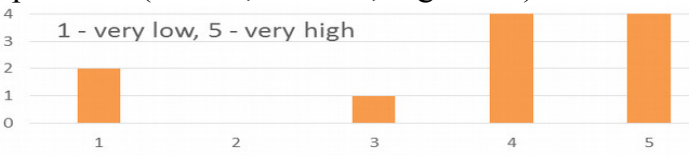

Figure 17 Usefulness of similar educational apps for learning with tablets

10. What screen size would you recommend for convenient use of such apps on mobile devices?

Most users preferred 10" screens for similar apps (Figure 18) 


\section{What screen orientation do you prefer when using the app with a smartphone/tablet?}

The testers preferred horizontal orientation when using the app with a tablet, and vertical with a smartphone (Figure 19).

\section{What caused problems?}

The main problem was learning to control the character, with the lack of noticeable controllers. The readability of information screens, texts on the floor and doors was low, Polish diacritics were missing, font sizes too small, and bold font unnecessary. The contrast between font colours and the background was insufficient. The text in the intro was too long, and displayed too quickly. It was not clear, which elements are interactive, and how to interact (approach or touch the element). Some testers had problems with door opening, or starting video. One person reported low level of graphics and non-intuitive introduction.

\section{What did you like best in the app?}

The users liked the clear arrangement of rooms, game environment, and setting the scientific issues in the space.

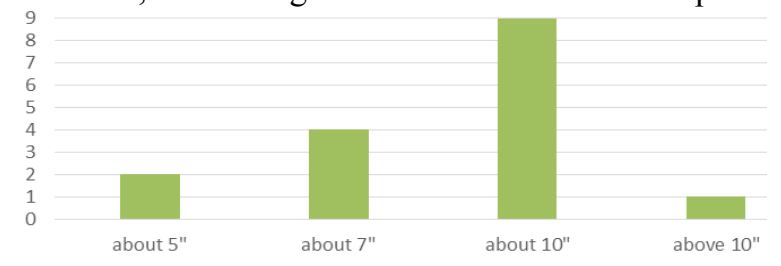

Figure 18 Screen size for similar apps (multiple choice question)

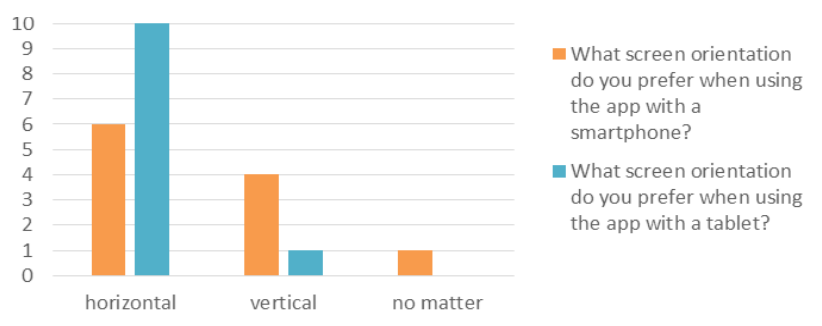

Figure 19 Screen orientation preferred o a smartphone or a tablet

\section{What should be improved in the app for tablets?}

The testers suggested more animations, decreasing the character's inertia, levelling of the camera view at eye level, and decreasing the transparency of the controllers. They also asked for bigger fonts, Polish diacritics, and different colours to improve contrast. Other suggestions:

- Adding an option to choose subtitles, or the narrator,

- Adding the button for skipping video material,

- Adding icons representing interactive elements,

- Better explanation of the goal of the game,

- Adjusting the scientific level to the target group.

15. What was missing in the app?

The users suggested to add interactive tasks, control the character perspective through a gyroscope (rotate the device) or gestures on touchscreens, and mark interactive spots.

\section{Other remarks}

The users asked for more interaction to enliven the game; the tasks should be more complex and engaging. The speech synthesizer (computer's voice) should be abandoned.

\section{SUMMARY}

We designed an edutainment app for learning physics on mobile devices, as young people commonly use them. The results of the usability tests of Apollo show that although some elements could be improved, the methodology applied to create it was properly selected.

\section{REFERENCES}

[1] L. Low, "M-learning standards review report", Australian Gov., 2007

[2] M. Ally, M., A. Tsinakos, (Eds.). "Increasing Access through Mobile Learning”. Commonwealth of Learning, Vancouver, 2014

[3] L. Stanislaus, J. Joseph (Eds.). Communication as Mission. Ishvani Kendra, 2007

[4] T. Heick, "9 Characteristics of $21 \mathrm{st}$ Century Learning". TeachThought, 2012

[5] K. Ayad, D. Rigas, "Learning with edutainment: A multi-platform approach", 11th MMACTEE, pp. 220-225, 2009

[6] W.J. Brown, A. Singhal, "Entertainment-Education Media Strategies for Social Change: Promises and Problems". In: D. Demen II, K. Villnnmath (Eds.): Mass Media. Social Control and Social Change: A macrosocial perspective. Iowa State University Press, 1999

[7] International Data Corporation, https://www.idc.com/, 2017

[8] Internet Live Stats, http://www.internetlivestats.com/, 2017

[9] K. Szklanny, M. Wichrowski, A. Wieczorkowska, „Educational App for Android Mobile Devices", Proc. AMCSE 2015, pp. 184-188

[10] Statista, The statistics portal https://www.statista.com/, 2017

[11] Ericsson, https://www.ericsson.com/, 2017

[12] J. Titcomb, "Mobile web usage overtakes desktop for first time", The Telegraph, 1 November 2016

[13] Impact, https://www.impactbnd.com/, 2017

[14] Bennett Coleman \& Co., Gadgets Now, www.gadgetsnow.com/, 2017

[15] CISCO., Gen y: New dawn for work, play, identity, http://www.cisco.com/, 2015

[16] T.T. Ahonen, "Updating Progress on the 'Recreate iPhone of 2010 for $\$ 10$ by 2020' Projection", Communities Dominate Brands, 2015

[17] J. Brown, "Future mobile learning", http://www.slideshare.net/, 2011

[18] Noteslate, http://www.noteslate.com/, 2016

[19] LG Electronics. http://www.lg.com/us/lg-g-flex-phones, 2017

[20] C. Carter, "New mobiles that change shape are 'only a matter of time'. The Telegraph, 29 April 2013

[21] Gartner, http://www.gartner.com/, 2017

[22] TNS, http://www.tnsglobal.com/, 2014

[23] Gemius, http://www.gemius.pl/, 2015

[24] TestMy Net, http://testmy.net/country/pl, 2017

[25] EducationalAppStore, http://www.educationalappstore.com, 2017

[26] K. Szklanny, M. Wichrowski, Prototyping M-Learning Course on the Basis of Puzzle Learning Methodology Learning and Collaboration Technologies. HCII 2014, LNCS 8524, 215-226, 2014

[27] Y. Hadzigeorgiou, "Humanizing the teaching of physics through storytelling: the case of current electricity". Phys. Educ. 41(1), 42, 2006

[28] P. Kokkotas, A. Rizaki, K. Malamitsa, ,Storytelling as a Strategy for Understanding Concepts of Electricity and Electromagnetism". Interchange 41(4), 379-405, 2010

[29] Unity, https://unity3d.com/, 2017

[30] Epic Games, What is Unreal Engine, www.unreal.com, 2017

[31] AUTODESK, 3ds Max. https://www.autodesk.com/, 2017

[32] Adobe, http://www.adobe.com/, 2017

[33] Quixel, http://quixel.se/, 2017

[34] IVONA, https://www.ivona.com/, 2017

[35] J. Nielsen, "Why You Only Need to Test with 5 Users". Nielsen Norman Group, 2000 